\section{Política de informação em saúde ambiental}

\section{Information policy in environmental health}

\section{Lia Giraldo da Silva Augusto}

Núcleo de Estudos em Saúde Coletiva-NESC

Centro de Pesquisas Aggeu Magalhães-CPqAM

Fundação Oswaldo Cruz-FIOCRUZ

Rua Ministro Nelson Hungria 266 apto. 201

Recife, PE CEP 51020-100

giraldo@cpqam.fiocruz.br

\section{Alice Branco}

Núcleo de Estudos em Saúde Coletiva-NESC

Centro de Pesquisas Aggeu Magalhães-CPqAM

Fundação Oswaldo Cruz-FIOCRUZ

\section{Resumo}

É imprescindível uma política de informação em saúde ambiental capaz de monitorar as políticas públicas, os processos produtivos e todas as atividades econômicas, bem como as ações de intervenção que visem melhorar as condições ambientais e de saúde das populações. Este artigo busca discutir os princípios norteadores de uma política de informação em saúde ambiental que permite monitorar o desenvolvimento sustentável. Para tal as autoras partem de uma revisão bibliográfica referente às questões conceituais e controversas do desenvolvimento sustentável e às perspectivas metodológicas para o desenvolvimento de uma política de informação em saúde ambiental. Para as autoras, além da necessidade de se criar sistemas e serviços de informação e comunicação que proporcionem o alerta $\mathrm{e}$ o suporte indispensáveis às políticas intersetoriais, é fundamental que estas incorporem os elementos das ações sociais e da participação das comunidades na definição dos indicadores voltados para o monitoramento do desenvolvimento humano.

Palavras-chave: Política de informação. Saúde ambiental. Desenvolvimento sustentável. Indicadores. 


\section{Abstract}

It is essential to have an information policy in environmental health capable of monitoring public policies, the productive processes and all the economic activities, as well as intervention actions that aim to improve environmental conditions and the health of populations. This article seeks to discuss the key principles of an information policy for environmental health that allows monitoring sustainable development. To this end, the authors reviewed references related to the conceptual questions and controversies of sustainable development and the methodological perspectives for the development of an information policy for environmental health. According to the authors, in addition to creating information and communication systems and services that provide attentive and indispensable support to intersectorial policies, it is crucial that these policies include the elements of social actions and the participation of the communities in the definition of pointers oriented towards monitoring human development.

Key words: Information policies. Environmental health. Sustainable development. Indicators.

\section{Introdução}

A legislação brasileira hoje em vigor apresenta aspectos importantes para o planejamento, a avaliação e o controle de condições nocivas no âmbito dos processos econômicos, sociais, do desenvolvimento técnico-científico e do ambiente físico. Tais instrumentos legais deveriam ser utilizados no sentido de favorecer sistemas de gerenciamento da qualidade de vida e da saúde.

Muito se fala em desenvolvimento sustentável e o discurso não é homogêneo; ele está marcado e é diferenciado em função dos interesses ambientais de diversos setores, grupos e atores sociais envolvidos no processo de desenvolvimento.

Mais que um conjunto de metas bem específicas, é um processo que implica em modificações econômicas e sociais. Uma "estratégia para promover a sustentabilidade é a importância da participação local e a revisão da forma como as pessoas vivem e trabalham"'.

Para fins deste artigo vamos conservar a definição de desenvolvimento sustentável dada pela Agenda 21: “... trata do desenvolvimento humano que conjuga e harmoniza o desenvolvimento social e econômico com democracia, liberdade e preservação dos recursos naturais disponíveis para a humanidade como um todo e para as futuras gerações"2.

Embora, este conceito pareça bastante amplo e consensual, a Agenda 21 vive a contradição dada pelo processo de globalização, entendida em geral como um processo de internacionalização e integração do capital.

Com a revolução científico-tecnológica dos meios de informação, comunicação e dos processos produtivos, há também profundas transformações políticas, sociais e culturais entre os povos, criando novas articulações entre o local e o global, configurando uma nova geopolítica mundial. Esse processo vem se dando como um movimento paradoxal de fragmentação territorial e de desintegração política ${ }^{3}$.

A "globalização" que hoje orquestra a economia do mundo, num processo de in- 
tensificação histórica da internacionalização dos mercados, tem obstaculizado o movimento na direção da diversidade ${ }^{4}$. Os poderosos grupos econômicos têm feito o possível para romper com as iniciativas individuais, locais e regionais, buscando modelarnos dentro de grupos sociais mais homogêneos e controláveis, do ponto de vista dos padrões de consumo, e, para tal, buscam influenciar nosso estilo de vida e interferir no ambiente entendido como dinâmico (histórico) e que é socialmente construído ${ }^{5}$.

A questão do desenvolvimento sustentável é, pois, controversa. Hoje há duas correntes de pensamento, uma que defende o processo de globalização e outra que busca resistir a ele. Fato curioso é que, recentemente, esses movimentos manifestaram seus pensamentos em paralelo. Para o Fórum Econômico Mundial, a globalização é um processo irreversível e ignora completamente a natureza e a dinâmica do processo gerador de desigualdades sociais. A outra tendência, expressa no Fórum Social Mundial $^{6}$, é de resistência e aposta na humanização do sistema, que deve evoluir em direção ao que Amartya Sen chama de "desenvolvimento com liberdade", muito além do mero crescimento econômico ${ }^{7}$.

A Agenda 21, para ser efetivamente um produto de consenso entre os diversos setores da sociedade, precisa tornar evidente para o conjunto da sociedade a sua inserção no contexto das temáticas do dia-a-dia das populações, seja nos centros urbanos, seja no campo. Da mesma forma, torna-se igualmente necessária à ampliação dos canais da participação popular e de controle social, para que a implantação da Agenda 21 no nível local seja legitimada como política intersetorial.

Essa luta pela inserção implica em se considerar as condições de dignidade no habitar, na atenção à saúde, na educação, no abastecimento de água, no esgotamento sanitário, no transporte, no acesso à alimentação adequada, nas adequadas relações de trabalho, no lazer, na atividade política, enfim, nos espaços de desenvolvimento humano. Entretanto, o nível "ideal” de dignidade não é e não deve ser o objeto central da discussão: o foco das atenções precisa estar voltado para as condições reais de vida de pessoas e grupos que, de um lado, pelo excesso e, de outro, pela carência absoluta, produzem uma sociedade em que a humilhação, o ressentimento, a arrogância e a falta de solidariedade levam a situações extremas de violência e de degradação da condição humana ${ }^{8}$ e do meio ambiente.

A garantia do desenvolvimento sustentável em área de intervenção produtiva, de tecnologias sofisticadas e de alto risco ambiental, requer dos investidores e dos governos práticas de planejamento estratégico, utilizando-se metodologias que dêem conta da complexidade dos sistemas e subsistemas neles envolvidos, direta ou indiretamente.

Nesse sentido, torna-se imprescindível uma política de informação em saúde ambiental capaz de monitorar as políticas públicas, os processos produtivos e todas as atividades econômicas, bem como as ações de intervenção que visem melhorar as condições ambientais e de saúde das populações.

Da mesma forma, a participação das comunidades e das organizações da sociedade civil é condição necessária para imprimir a esse processo um caráter de sustentabilidade.

Esse artigo busca discutir os princípios norteadores de uma política de informação em saúde ambiental que permita monitorar o desenvolvimento sustentável.

\section{A perspectiva sistêmica}

Na compreensão da Saúde Coletiva, o diagnóstico de uma determinada situação deve estar centrado na identificação de processos e mecanismos que estão integrados numa cadeia de eventos interdefiníveis e que são historicamente determinados. O que acontecerá no futuro e o que acontece hoje é mais bem interpretado em termos da história e o diagnóstico situacional implica diversos níveis de processos:

- No primeiro nível estão as modificações que afetam o meio ambiente físico, as 
relações sócio-econômicas e a população da área de abrangência e suas condições de vida. Em geral, estão relacionadas com modificações no sistema produtivo da região.

- No segundo nível estão as modificações introduzidas pelos processos de industrialização e de pólos de desenvolvimento que resultam em processos significantes do primeiro nível.

- No terceiro nível estão, entre outros: as modificações nas políticas de desenvolvimento nacional, os mercados, os fluxos de capital, que por sua vez determinam modificações no segundo nível.

Cada um desses três níveis tem sua própria dinâmica e, conseqüentemente, requer dados e escalas específicos para sua análise.

A produção de informação em saúde ambiental deve ter como objetivo a análise de possíveis cenários e ser complementada por avaliações das tecnologias em execução e das possibilidades de mudanças, adaptações e inovações para adequá-las aos problemas específicos a serem resolvidos, a partir dos recursos existentes no meio físico, social, econômico e dos interesses locais e nacionais que podem ser favorecidos ou afetados negativamente, bem como das possibilidades oriundas do contexto internacional.

Diagnósticos baseados exclusivamente na verificação de certos parâmetros, num dado momento, são insuficientes para afirmar a "sustentabilidade" do sistema produtivo ou a garantia da qualidade de vida.

\section{Fundamentos para uma política de informação em saúde ambiental}

A informação em saúde ambiental pode contribuir para melhorar a gestão e as políticas "sanitárias" em todos os países do mundo, mas é particularmente valiosa para os países onde a questão da degradação ambiental tem ocupado um lugar secundário frente às exigências do desenvolvimento econômico, imposto de fora pela globalização e pelas políticas neoliberais. É certa- mente importante para impor limites ao processo de espoliação ambiental e da saúde das populações.

Evidentemente, os gestores necessitam de informação confiável e pertinente sobre a gravidade dos problemas ambientais e suas relações com a saúde humana. Por conseguinte, têm sido feitos muitos esforços para estabelecer indicadores que sirvam de base para as medidas normativas e gerenciais. Mas existe sempre o perigo de que sejam alimentadas expectativas demasiadas sobre o que se pode esperar de um sistema de informação, e por isto é preciso deixar claro que a informação não é uma panacéia.

Trata-se de um recurso cuja gestão obedece, inevitavelmente, ao conjunto de interesses predominantes em cada setor específico de atuação, subordinada à política vigente para o mesmo.

Desta forma, para minimizar os aspectos da desigualdade de poder na definição das políticas convém instituir um processo amplo de negociação para que os diversos interesses se explicitem e seja possível um sistema que ao menos sirva aos propósitos constitucionais definidos para o Sistema Nacional de Saúde.

A política de informação pode ser definida como o conjunto de leis, regulamentos, decisões, ações e interesses que orientam a geração, circulação, tratamento, armazenamento e uso da informação.

Como política pública, assenta-se sobre interesses e metas políticas e burocráticas, não necessariamente congruentes, manifestando-se para além do aparato governamental. Expressa-se não apenas no campo formal das leis e regulamentos, mas também no informalismo das práticas e ações. Afinal, como qualquer política, é sempre exercida num contexto em que se misturam pessoas, instituições e interesses, cujas manifestações nem sempre se revelam por mecanismos formais ${ }^{9,10}$.

É nesse sentido que a política de informação deve ser exercida como "arte de compromisso", ou seja, como capacidade de estabelecer compromissos entre interesses contraditórios e por vezes antagônicos, não 
podendo ser classificada a priori como boa ou má11. Uma vez que se assenta sobre o permanente conflito entre atores, objetivos, metas, interesses, planos e ações, que não pode ser completamente resolvido em favor de qualquer pólo envolvido, mas que reflete claramente a quê (ou a quem) serve, sendo este um aspecto a ser reconhecido e refletido criticamente, para permitir avanços, em especial se o objetivo é o desenvolvimento humano, e nesse sentido a questão ética deve ser ressaltada.

Trata-se, pois, de um processo dinâmico, flexível e responsivo à mudança de circunstâncias e cenários, sendo difícil prever seu resultado ou impacto, e obriga a lidar com os limites impostos por essa incerteza.

No caso brasileiro, a pouca articulação entre as distintas esferas de atuação do Estado, particularmente na área social, tem gerado uma política de informação pouco integradora. O que se verifica são políticas setoriais de informação que desvinculam temas como saúde, educação, trabalho e meio ambiente, dentre outros.

Empobrecida pela visão compartimentalizada, a informação produzida pelas bases de dados e sistemas de informação dessas áreas dificulta sua utilização como subsídio, quer para a tomada de decisão, quer para a ação social. As interfaces entre os problemas que se originam nesses diferentes setores não são explicitadas pela informação assim gerada.

Isso porque cada uma dessas bases e sistemas é estruturada segundo esquemas interpretativos próprios, arbitrados segundo o que se deseja informar. São estabelecidos limites para o registro de eventos, que vão desde grupos populacionais até áreas geográficas, recortando ainda mais o que, em essência, é indivisível: os fatos e seu contexto.

A justificativa para tanto costuma ser a de que é impossível abarcar a totalidade de relações ou de condições de um dado fenômeno, o que obriga a selecionar alguns aspectos do entorno. Contudo, tal seleção pode se dar mediante critérios mais integradores e contextualizadores, fundamentados por uma ética social. Mais do que se preocupar com as respostas que a informação poderá gerar propõe-se inverter a lógica e refazer as perguntas; formular os problemas para além do próprio campo de atuação, identificando os processos e mecanismos que a eles conduziram.

Piaget \& García ${ }^{12}$ já demonstraram que o conhecimento científico tem progredido mais pela contribuição daqueles que foram capazes de formular novas perguntas para antigos problemas do que por encontrar novas respostas para velhas perguntas.

É claro que os dados disponíveis nos diferentes setores governamentais podem ser usados para análises relacionais sobre a saúde ambiental, mas somente a ampliação do marco epistêmico, político e social sobre o qual repousa essa produção de informações poderá gerar uma política de informação inclusiva.

Uma pergunta implícita em nosso objetivo é se uma política de informação para o projeto de desenvolvimento sustentável teria como objetivos apoiar as ações de controle para deter ou reverter a deterioração do ambiente; para desenvolver a qualidade de vida da população ou para estabelecer condições de estabilidade social, econômica e ambiental.

O que se pode dizer é que esses objetivos não se referem a um estado do sistema, mas a processos, que são sucessões temporais de eventos inter-relacionados, em seqüências causais e de eventos não-observáveis, posto que os processos não são observáveis, são resultado das interferências dos observáveis.

Assim, as relações que determinam os processos constituem uma estrutura sistêmica formada pela situação social, econômica e ambiental que apresenta problemas que estão ligados às propriedades estruturais do sistema (fragilidade, vulnerabilidade e resistência) ${ }^{13}$.

Os períodos críticos na evolução de um sistema apresentam-se quando os processos de deterioração excedem a resistência da estrutura ou quando o sistema está desestabilizado por fortes flutuações. A vulnerabilidade de um sistema não é distri- 
buída uniformemente através de sua estrutura. Não ocorre instabilidade simultaneamente em todos os subsistemas. A ruptura do sistema começa em áreas específicas. Aqui reside a importância de um sistema de informação para a saúde ambiental voltada para a monitoração do desenvolvimento que se quer sustentável.

Uma política de informação para a sustentabilidade é fundamental. Se considerarmos que os indicadores epidemiológicos, por exemplo, servem para indicar apenas aspectos dessa ruptura, vemos claramente que a avaliação sistêmica de um projeto de desenvolvimento sustentável deveria priorizar a análise da evolução dos processos que estão ligados às suas propriedades estruturais.

Com base nesses pontos, é possível estabelecer que observações, medidas, análises e informações serão necessárias para se inferir os processos que estão vinculados à deterioração social, econômica e física do ambiente, e aqueles que estabilizam ou desestabilizam o sistema. Para essa monitoração, tanto precisamos de estudos quantitativos, que usam um sistema numérico de medidas, como de estudos qualitativos, que usam sistemas lógicos de linguagem (simbólicos). Os resultados desses estudos são dados empíricos cuja interpretação origina os observáveis. Portanto, conclui-se que não há observáveis "puros"; eles estão carregados de subjetividade. Como apreender essa subjetividade é uma tarefa de criatividade a que alguns pesquisadores, em especial das correntes de pensamento construtivista, têm se proposto ${ }^{14-17}$.

Nas últimas décadas tem crescido a preocupação com a busca de indicadores de qualidade de vida e saúde ambiental, dando origem a iniciativas de organização e sistematização de dados e informações sobre o tema.

Em 1993, o conceito de indicador ambiental foi definido pela Organization for Economic Cooperation and Development $(\mathrm{OECD})^{18}$, que para tal propôs um modelo denominado em português FPEEEA (Forças Motrizes/Pressão/Estado/Exposição/Efei- tos/ Ação), que sistematiza as principais etapas do processo de geração, exposição e efeitos dos riscos ambientais e das ações de controle, prevenção e promoção referentes a cada etapa.

A Organização Mundial da Saúde (OMS $)^{19}$ apoiou-se nesse modelo, adaptando-o para a saúde e indicando-o como um instrumento que facilita a compreensão das relações entre saúde e ambiente, e como subsídio para o desenvolvimento de ações e a tomada de decisão.

O Ministério da Saúde, por meio da Fundação Nacional de Saúde, entende que o modelo FPEEEA “... revela a necessidade de serem integradas as análises dos efeitos dos riscos ambientais para a saúde das populações, com o desenvolvimento e implementação de processos decisórios, políticas públicas e práticas de gerenciamento de ris$\cos ^{\prime 20}$.

O FPEEEA fundamenta-se em seis grandes categorias de análise:

- Forças Motrizes, que representam o modelo de desenvolvimento adotado, responsável pelas atividades e fontes de poluição e de degradação ambiental;

- Pressão, que corresponde às fontes de pressão sobre o ambiente e sobre as populações, estando subordinadas às forças motrizes;

- Estado, que diz respeito às condições ambientais gerais submetidas às pressões existentes;

- Exposição, que se refere aos riscos produzidos à saúde ambiental e humana;

- Efeitos, que são o resultado nocivo da submissão à exposição; $\mathrm{e}$

- Ações, que são as medidas de proteção e promoção e proteção da saúde humana e do ambiente.

Os indicadores de saúde ambiental têm sido incorporados às atividades de vários programas da OMS. A primeira reunião para tratar especificamente do tema ocorreu em $1992^{19}$. Desde então, vêm sendo realizados vários encontros para debater o desenvolvimento de indicadores, como por exemplo, os referentes ao projeto de análise sanitária e ambiental para a tomada de decisões ou 
para a iniciativa de montagem de sistema de informação geográfico em saúde ambiental.

Também a Organização das Nações Unidas $(\mathrm{ONU})^{22}$ tem promovido e incentivado uma série de ações internacionais no âmbito do Plano de Ação Global. Dentre os projetos que vêm sendo executados encontrase o Programa de Indicadores, iniciado em 1994 e em desenvolvimento em cerca de 110 países. O objetivo é criar uma base única de indicadores-chave globais de saúde ambiental e qualidade de vida que possa vir a proporcionar informação consistente e comparável $^{22}$.

A construção de indicadores tem sido um desafio de importância fundamental, pois o indicador faz parte da semântica da informação e não pode ser apresentado como um ente isolado.

$\mathrm{Na}$ verdade, o indicador deve ser um revelador da complexidade do problema ${ }^{23}$. A representação da realidade, no sentido da compreensão de um dado fenômeno a ela pertencente exige que o "recorte do objeto" ou o "objeto modelo", do qual faz parte o indicador, deve ter uma "pré-compreensão modelizante", que é a maneira como se pode estudar a realidade e validá-lo. Como de alguma outra forma já foi dito, os indicadores não devem ser apropriados como um valor neutro. Um indicador deve compor uma informação que precisará de uma linguagem capaz de comunicar.

Segundo Barcelos ${ }^{24}$, do ponto de vista dos sistemas de informação, as condições ambientais são atributos do lugar, enquanto as condições de saúde são atributos das populações humanas. No entanto, estes processos são interdefiníveis e por esta razão exigem uma abordagem integrada.

A triangulação metodológica constitui a chave para a análise dos problemas sócioambientais, que são de natureza complexa, e para resolver a validação dos indicadores compostos, uma vez que eles não estão relacionados às condições específicas e não estão resumidos às operações de mensuração quantitativa ${ }^{25}$.

O problema dos sistemas de informação não está, pois, limitado na competência de operar diversas variáveis e indicadores, mas na capacidade de triangulação dos dados, tanto do ponto de vista quantitativo como qualitativo, e levar em consideração tanto sua verticalidade (hierarquia, ordenação) como horizontalidade (redes, coordenação); isto só é possível numa abordagem dialética ${ }^{26}$ e dialógica ${ }^{27}$.

Os indicadores refletem valores coletivos e sua legitimação não se dá por ensaios técnicos, do tipo simulação virtual. Trata-se de um processo vivo que propomos leve em consideração toda a subjetividade que, segundo Guattari ${ }^{28}$, funciona como uma ecosofia, onde a estética, a ética e a subjetividade se apresentam como entes reais a serem considerados e reconhecidos na estrutura técnica da informação para que os indicadores cumpram seu objetivo de comunicar.

\section{Conclusões}

Para o Brasil, detentor de ricas e estratégicas reservas naturais, a lógica do desenvolvimento sustentável deve constituir referência básica para a política de informação ambiental do país. É sob a perspectiva da sustentabilidade ecológica e social que as técnicas, metodologias e tecnologias do campo da informação devem ser geridas, de forma a permitir melhor conhecer, diagnosticar e monitorar as condições ambientais, sobretudo em função da extensão do território nacional, de sua biodiversidade, da diversidade cultural e complexidade dos problemas ambientais, sociais e econômicos envolvidos.

Cabe à política de informação ambiental criar sistemas e serviços de informação e comunicação que proporcionem o alerta $\mathrm{e}$ o suporte indispensáveis não apenas às políticas públicas, mas também às ações sociais. Com as novas mídias e redes eletrônicas criam-se também condições mais favoráveis para a informação e conscientização políticas no que se refere ao meio ambiente e à educação ambiental, facilitando a implantação de um modelo mais sustentável de desenvolvimento. 


\section{Referências}

1. Leff, E. Educación en Ambiente para el Desarrollo Sustentable. Buenos Aires: Ed. Escuela Pedagógica y Sindical "Marina Vilte" de CTERA, 1998.

2. Agenda 21Brasileira - bases para discussão. Brasília, DF: Ed. MMA, PNUD; 2000.

3. Rattner H. Salvar quem, cara pálida? São Paulo: ABDL; julho de 2001. rattner@abdl.org.br

4. Rattner H. Meio ambiente e desenvolvimento sustentável: o mundo na encruzilhada da História. Texto elaborado como contribuição à discussão preparatória da Conferência das Nações Unidas sobre Meio Ambiente e Desenvolvimento Sustentável, agendada para a gosto-setembro de 2002, em Johannesburgo, África do Sul; 2002. Disponível em <http://www.abdl.org.br/Rattner/inicio.htm>

5. Santos M. O retorno do território. In: Santos M, Souza MAA, Silveira ML. Território, Globalização e Fragmentação; $4^{a}$ ed. São Paulo: Ed. Hucitec; 1998. p.15-20.

6. Rattner H. Fórum Social Mundial X Fórum Econômico Mundial: Existem Alternativas? São Paulo: Ed. Associação Brasileira para o Desenvolvimento de Lideranças; 2002. Disponível em <http:// www.abdl.org.br>; rattner@abdl.org.br.

7. Sen A. On Economic Inequality. Expandide edition by James E. Foster \& Amartya Sen. New York: Oxford University Press; 1997.

8. Conselho Nacional de Saúde-CNS. A saúde na Agenda 21 brasileira. Proposta da oficina organizada pelo CNS, OPS, FIOCRUZ, ABRASCO. Brasília, 26 e 27 de julho de 2001.

9. Browne M. The field of information policy: 1 . Fundamental concepts. Journal of Information Science 1997; 23(4): 261-75.

10. Eisenchitz T. Information Transfer Policy. London: Library Association; 1993.

11. Piaget J, García R. Psicogénesis e Historia de la Ciencia. México: Siglo XXI; 1982.

12. Rowlands I. Understanding information policy: concepts, frameworks and research tools. Journal of Information Science 1996; 22(1): 13-25.

13. Garcia R. From Planning to Evaluation. A systems approach to sustainable development projects. México: Ed. CINVESTAV; 1996.

14. Morin E. O Método. Tomo II, São Paulo: Ed. EuropaAmérica; 1996.

15. Simon HA. Complexity and the representation of patterned sequences of symbols. Republicado in Models of Thought. New Haven: Yale University Press; 1979 .
16. Piaget J, Garcia R. Hacia uma Logica de Significaciones. Cap. III. Barcelona: Ed. Gedisa; 1987.

17. Guattari F. Caosmose: um novo paradigma estético. $3^{\mathrm{a}}$ Ed. Coleção Trans. São Paulo: Editora 34; 2000.

18. Organização para Cooperação e Desenvolvimento Econômico-OCDE. Core set of indicators for environmental performance review. Environmental Monography, n.83. Paris: Ed. OCDE; 1993.

19. Will J, Briggs D. Developing Indicators for Environment and Health. World Health Statiscs Quarterly 1995; 48(2):155-63.

20. Fundação Nacional de Saúde-FUNASA. Curso Básico de Vigilância Ambiental em Saúde (CBVA). Módulo III - Instrumentos Básicos para a Vigilância Ambiental em Saúde. Brasília: Ministério da Saúde. Ed. FUNASA/MS; 2000 .

21. Yassi A, Kjellström T, De Kok T, Guidotti T. Salud Ambiental Básica. Série Textos Básicos para la Formación Ambiental. México: Ed. PNUMA, OMS, INHEM; 2002.

22. Forge, I. Información e Indicadores Ambientales Urbanos. In. Comissión Económica para la America Latina y el Caribe.Washington DC: World Health Statiscs Quarterly Vol. 48 (2):155-163, 1995.

23. Galvão LA, Oliveira MLC, Augusto LGS et al. Indicadores de Saúde e Ambiente - relatório da Oficina de Trabalho realizada durante o IV Congresso Brasileiro de Epidemiologia. Epi-RIO-98. Informe Epidemiológico do SUS 1998; 7(2): 46-53.

24. Barcelos C. Constituição de um Sistema de Indicadores Socioambientais. In: Minayo MCS, Mirando AC. Saúde e Ambiente Sustentable: estreitando nós. Parte V2. Rio de Janeiro: Ed. Abrasco/FIOCRUZ; 2002.

25. Augusto LGS. A construção de Indicadores em Saúde Ambiental: desafios conceituais. In: Minayo MCS \& Mirando, AC. Saúde e Ambiente Sustentable: estreitando nós. Ed. Abrasco/FIOCRUZ. Parte V1, 2002.

26. Samaja J. La Semantica del discurso científico y el analisis de Matrices de Datos. Buenos Aires. 43p.Texto Preparado para a Disciplina "Saúde, Ambiente e Trabalho”, do Curso de Mestrado em Saúde Pública do Centro de Pesquisas Aggeu Magalhães/FIOCRUZ. Recife, 25-29/10/1999 (mimeo).

27. Pena-Vega A. Perception des risques, Qualité de vie et Ethique. Thèmes et Séances. Paris; 2001 (mimeo).

28. Guattari F. Caosmose: um novo paradigma estético. $3^{a}$ ed. Coleção Trans. São Paulo: Editora 34; 2000.

Recebido em: 24/07/2002 Aprovação em: 29/10/2002 